\title{
ESPLORANDO "FLATLANDIA": DAL GRAFENE ALLA SCOPERTA DI NUOVI MATERIALI BI-DIMENSIONALI
}

\author{
FRANCESCO TUMINO (*)
}

Nota presentata dal m.e. Carlo Bottani

(Adunanza del 16 novembre 2017)

SuNTO. - L'avvento delle nanotecnologie ha stimolato la ricerca verso nuovi nanomateriali per applicazioni innovative. Nel 2004, la produzione e lo studio del grafene ovvero un singolo strato di atomi di carbonio - mise in luce, per la prima volta, le eccezionali proprietà di questo materiale e aprì la strada alla ricerca sui cosiddetti materiali bi-dimensionali (2D). Da allora, molti nuovi materiali 2D sono stati prodotti e studiati, evidenziando proprietà e comportamenti nuovi rispetto alle più note versioni "3D" degli stessi materiali. La ricerca sui materiali 2D è oggi uno dei settori più attivi e promettenti della scienza dei materiali e pone le basi per lo sviluppo di nuove tecnologie, ad esempio, nel campo dell'elettronica, dell'optoelettronica, dell'energia e della sensoristica molecolare. In questo articolo, verranno introdotti alcuni importanti aspetti di questa ricerca - come i metodi di sintesi e le tecniche di caratterizzazione dei materiali $2 \mathrm{D}$ - e verranno brevemente discusse alcune caratteristiche generali di questi materiali alla luce di recenti studi sperimentali effettuati tramite la tecnica di microscopia a effetto tunnel (STM).

$$
* * *
$$

ABSTRACT. - The development of nanotechnology has encouraged the research of new nanomaterials for innovative applications. In 2004, the production and study of graphene - that is a single layer of carbon atoms - showed, for the first time, the extraordinary properties of this material and opened the way to the exploration of the so-called two-dimensional (2D) materials. Since then, several 2D materials have been

(*) Dipartimento di Energia, Politecnico di Milano, Italy.

E-mail: francesco.tumino@polimi.it 
produced and studied, revealing properties and behaviours, in general, very different from those of corresponding bulk materials. Research on 2D materials is nowadays one of the most active and promising fields of materials science, which is setting the basis for the development of novel technologies, such as in electronics, optoelectronics, energy and molecular sensing. In this paper, some important aspects of the study of $2 \mathrm{D}$ materials will be introduced - such as the synthesis methodologies and characterization techniques - and some of their properties will be shown, with the support of recent experimental results of scanning tunneling microscopy (STM) investigations.

\section{INTRODUZIONE}

La scoperta di nuovi materiali ha sempre avuto, dalla preistoria fino ai giorni nostri, un impatto enorme sul progresso tecnologico dell'uomo. In tempi recenti, la ricerca di nuovi materiali e il conseguente sviluppo di nuove tecnologie hanno ricevuto forte impulso, soprattutto grazie ai grandi passi avanti compiuti nella chimica e nella fisica della materia nel corso del XX secolo. Tale sviluppo ha permesso la nascita delle nanotecnologie, che si basano essenzialmente sulla manipolazione della materia su scala dimensionale dell'ordine del nanometro. Uno degli aspetti più innovativi di questo approccio risiede nella possibilità di accedere alle diverse proprietà che i materiali assumono quando le loro dimensioni sono ridotte fino alla scala nanometrica. La dimensione, infatti, gioca un ruolo fondamentale nel determinare la struttura elettronica di un materiale, dalla quale dipendono le sue proprietà ottiche, elettriche, meccaniche, termiche, magnetiche, chimiche. I materiali bidimensionali (2D) forniscono un esempio evidente di come ridurre la dimensione spaziale di un materiale - una sola dimensione in questo caso - possa far emergere proprietà e comportamenti nuovi rispetto a quelli dello stesso materiale "tri-dimensionale". Si consideri, ad esempio, la grafite, che, come molti sanno, è un materiale molto comune formato da atomi di carbonio. Se immaginiamo di ridurre lo spessore di un campione di grafite fino ad isolare un solo strato atomico, otteniamo un nuovo materiale dalle proprietà uniche: il grafene. Lo studio del grafene valse il premio Nobel per la fisica nel 2010 a Andre Geim e Konstantin Novoselov [1], e aprì la strada alla produzione e allo studio di nuovi materiali $2 \mathrm{D}$, quali il nitruro di boro esagonale $(\mathrm{hBN})$, il disolfuro di molibdeno $\left(\mathrm{MoS}_{2}\right)$, il silicene e altri ancora [2]. Oggi, la ricerca sui materiali $2 \mathrm{D}$ e sulle strutture che si possono ottenere combinandoli tra loro è uno dei campi più attivi della scienza dei materiali, potenzialmente in 
grado di innovare radicalmente i futuri dispositivi elettronici e optoelettronici [3] [4]. Proprio come in Flatlandia - l'universo bi-dimensionale immaginato da Edwin Abbott Abbott nel suo racconto del 1884 - l'esplorazione del mondo dei materiali $2 \mathrm{D}$ si profila come un'importante occasione di estendere la nostra conoscenza e di concepire nuovi scenari per il futuro sviluppo tecnologico e sociale. Una descrizione esaustiva e dettagliata della scienza dei materiali 2D va, naturalmente, oltre lo scopo di questo articolo, che, piuttosto, intende fornire una breve e generale introduzione a questa classe di materiali (sezione 2), mettendo in evidenza aspetti importanti della ricerca, quali i possibili metodi per la loro fabbricazione (sezione 3) e tecniche per la caratterizzazione delle loro proprietà (sezione 4). A scopo esemplificativo, verranno anche mostrati alcuni recenti risultati sperimentali ottenuti tramite osservazione al microscopio a effetto tunnel (STM) di alcuni materiali 2D (sezione 4.1).

\section{Materiali ed eterostrutture 2D}

Come accennato nell'introduzione, i materiali 2D sono materiali "super-sottili", aventi uno spessore dell'ordine del nanometro. Appartengono, quindi, a questa categoria quei materiali composti da uno - come il grafene - o, al più, pochi strati atomici. La bassa dimensionalità di questi materiali conferisce loro proprietà, in generale, molto diverse da quelle delle versioni "3D" (o bulk) dei corrispettivi materiali. A parte il grafene, un altro evidente esempio di come il numero di strati atomici influenza le proprietà di un materiale è fornito da un'importante famiglia di materiali 2D: i dicalcogenuri di metalli di transizione (TMD). Il principale, e più studiato, rappresentante di questa famiglia è il disolfuro di molibdeno $\left(\mathrm{MoS}_{2}\right)$, che si trova comunemente in natura sotto forma del minerale molibdenite. Si tratta di un materiale simile alla grafite, in quanto formato da strati atomici debolmente legati tra loro (da interazioni di tipo van der Waals), ciascuno dei quali composti da atomi legati tra loro da legami molto forti, di tipo covalente. A differenza della grafite, il singolo strato di $\mathrm{MoS}_{2}$ ha, oltre alla composizione chimica, una struttura diversa, in quanto formato da tre piani atomici: un piano di atomi di molibdeno tra due piani di atomi di zolfo. Questo materiale, così come altri TMD (WS $, \mathrm{MoSe}_{2}, \mathrm{WSe}_{2}$, ecc.), si distingue per una peculiare modifica delle sue proprietà opto-elettroniche quando si passa da molti strati atomici a un solo strato atomico (cioè al limite bi-dimen- 
sionale). Tale modifica interessa, in particolare, la sua struttura elettronica e si manifesta nella transizione da materiale semiconduttore a gap indiretto (nel caso 3D) a semiconduttore a gap diretto (nel caso 2D). Dal momento che il gap energetico è nell'intervallo di luce visibile (vale 1.9 $\mathrm{eV})$, tale transizione implica che l'interazione con la luce - in termini, ad esempio, di assorbimento o fotoluminescenza - sia molto più intensa per il singolo strato di $\mathrm{MoS}_{2}$ rispetto alla versione multi-strato, caratteristica che fa di questo materiale $2 \mathrm{D}$ un promettente candidato come principale componente di futuri dispositivi opto-elettronici [5]. Un altro aspetto che rende interessante lo studio dei materiali $2 \mathrm{D}$ è il fatto di avere proprietà molto diverse tra loro, oltre che rispetto ai materiali bulk. Ad esempio, con riferimento alla struttura elettronica, troviamo semiconduttori a zero gap (il grafene), semiconduttori con gap nel visibile (il $\mathrm{MoS}_{2}$ ), metalli (il NbSe) e isolanti, come il nitruro di boro esagonale $(\mathrm{hBN})$. Quest'ultimo è uno dei materiali $2 \mathrm{D}$ tradizionalmente più studiati oltre il grafene, del quale possiede la stessa struttura a nido d'ape, nella quale, però, si alternano atomi di boro e azoto, anziché di carbonio. Ciò è sufficiente a conferire allo $\mathrm{hBN}$ proprietà molto diverse da quelle del grafene, come un ampio gap energetico $(6 \mathrm{eV})$. Esistono altri materiali 2D graphene-like, cioè con struttura simile a quella del grafene: di recente, ad esempio, sono stati studiati il silicene, il germanene e lo stanene (anche chiamati X-eni), ovvero le "versioni 2D" di silicio, germanio e stagno, rispettivamente. A questo punto è opportuno fare un'osservazione di carattere generale. Materiali 2D come grafene, $\mathrm{MoS}_{2}$ o hBN non sono altro che il singolo strato di una struttura cristallina a molti strati, ed è quindi possibile produrli separando tra loro i vari strati fino ad isolarne uno solo (tramite tecniche di esfoliazione, come descritto nella sezione 3). Invece, dal momento che materiali come silicio, germanio o stagno non possiedono una struttura stratificata, i corrispondenti materiali $2 \mathrm{D}$ non possono essere ricavati per esfoliazione della struttura bulk, ma vanno sintetizzati tramite tecniche in grado di far "crescere" un singolo strato atomico su un substrato adatto a questo scopo. In questo caso, l'interazione con il substrato è un parametro che contribuisce significativamente, insieme al confinamento dimensionale, a determinare le proprietà strutturali ed elettroniche del materiale 2D supportato. Questo aspetto è centrale per la sintesi di molti materiali 2D, compresa un'importante classe di questi materiali: quella degli ossidi $2 D$. I materiali ossidi si trovano abbondantemente in natura, sono economici, non tossici, dotati di grande stabilità chimica e termica e, per questo, utiliz- 
zati in moltissime applicazioni. Per la loro importanza, questi materiali occupano da decenni una posizione centrale nella scienza e tecnologia dei materiali. Di recente, lo studio di ossidi a bassa dimensionalità ha portato all'osservazione di nuove strutture $2 \mathrm{D}$, che possono trovare applicazione in ambiti tecnologici avanzati [6]. La vasta gamma di proprietà e funzionalità offerte dagli ossidi, estende ulteriormente lo spettro di potenzialità dei materiali 2D. La grande varietà che si osserva all'interno di questa classe di materiali ha fatto emergere, negli ultimi anni, un nuovo paradigma nella ricerca e nel design delle future applicazioni di questi materiali: combinare insieme diversi materiali $2 \mathrm{D}$ per creare ad arte dei materiali più complessi - le cosiddette eterostrutture $2 \mathrm{D}$ - con nuove proprietà e funzionalità. Questi nuovi materiali possono essere realizzati impilando uno sopra all'altro diversi materiali $2 \mathrm{D}$, a formare eterostrutture multi-strato, in cui, idealmente, ogni strato atomico corrisponde a uno specifico materiale 2D. Tali eterostrutture - solitamente chiamate eterostrutture di van der Waals, per via della natura del legame tra i vari strati — sono attualmente oggetto di un'intensa attività di ricerca sia di carattere teorico sia sperimentale, volta alla realizzazione di prototipi per lo studio sia delle loro proprietà fondamentali sia delle loro prestazioni in un'ottica più applicativa [7]. Naturalmente, l'obiettivo a lungo termine di realizzare dispositivi efficienti basati su eterostrutture 2D presuppone non solo uno sviluppo della conoscenza di base di questi sistemi, ma anche la ricerca di metodi per la loro fabbricazione che siano affidabili, scalabili e in grado di fornire un sufficiente grado di controllo sulle caratteristiche dei materiali prodotti.

\section{PRODURRE I MATERIALI 2D: METODI DI FABBRICAZIONE}

Storicamente, la prima tecnica messa a punto per ottenere il grafene fu la cosiddetta esfoliazione micro-meccanica. Questa tecnica prevede l'utilizzo di comune nastro adesivo per esfoliare ripetutamente un campione di grafite fino ad ottenere dei "pezzi" (flakes) sempre più sottili. Questa operazione produce anche dei flakes spessi uno strato atomico, cioè il grafene. Si tratta di una tecnica concettualmente molto semplice ed economica, che ha consentito di effettuare, nel 2004, i primi importanti esperimenti sul grafene e rivelarne le proprietà uniche. Successivamente, è stata applicata ad altri materiali esfoliabili, come $\mathrm{MoS}_{2}$ e hBN, permettendo di produrre nuovi materiali 2D oltre il gra- 
fene [8]. L'esfoliazione micro-meccanica è ancora oggi molto utilizzata nei laboratori di ricerca e, combinata a tecniche di trasferimento preciso dei flakes, può essere impiegata per la produzione di eterostrutture verticali di diversi materiali 2D. Tuttavia, questa tecnica presenta anche importanti limiti, tra cui quelli di non offrire nessun grado di controllo sullo spessore dei flakes ottenuti e di richiedere molto tempo per l'individuazione dei flakes mono-strato. Un passo avanti in questo senso è rappresentato dall'esfoliazione in fase liquida, ovvero l'esfoliazione del materiale bulk tramite specifici agenti chimici in soluzione. Tuttavia, è opportuno sottolineare che le tecniche di esfoliazione non consentono di produrre materiali 2D di elevata area superficiale e che non sono applicabili a materiali non esfoliabili, non permettendo, quindi, la produzione di molti materiali 2D la cui controparte bulk non sia formata da strati debolmente legati tra loro. Per produrre questi materiali - ad esempio il silicene - è necessario adottare un approccio differente, di tipo bottom-up, che consiste nel "crescere" il materiale a partire dai suoi costituenti elementari, atomi o molecole. Per fare ciò si possono utilizzare diverse tecniche di deposizione, che consentono di sintetizzare il materiale su substrati che ne favoriscano la crescita. Una delle tecniche di deposizione più investigate per la crescita di materiali 2D è la deposizione chimica da vapore (CVD), che permette di sintetizzare film di elevata qualità cristallina e ampia estensione (anche su scala del centimetro). In un tipico processo di CVD, uno o più precursori molecolari volatili vengono fatti reagire chimicamente in modo da produrre il film sul substrato. Quest'ultimo, al fine di promuovere le reazioni chimiche in gioco, viene tipicamente portato a temperature relativamente elevate (intorno a $1000{ }^{\circ} \mathrm{C}$, indicativamente). Essendo la temperatura un parametro critico nell'ottica di una produzione su larga scala dei materiali 2D, lo sviluppo di metodi di sintesi che richiedano una minore temperatura di processo è altamente desiderabile. Per questo motivo, altre tecniche di deposizione, alternative alla CVD, vengono studiate e ottimizzate per la produzione di materiali 2D. In particolare, le tecniche di deposizione fisica da vapore (PVD) consentono generalmente di produrre film ultra-sottili di diversi materiali con temperature di sintesi più basse rispetto alla CVD. Per PVD si intendono processi di deposizione nei quali il materiale viene evaporato tipicamente da una sorgente solida in forma di atomi o molecole e trasportato in forma vapore o plasma fino al substrato su cui condensa. Tra le tecniche PVD, è interessante menzionare la deposizione a impulsi laser (PLD), che si basa sull'abla- 
zione di un bersaglio solido da parte di impulsi laser ad alta energia. L'interazione laser-materia causa l'ablazione del bersaglio e la conseguente espulsione del materiale ablato sotto forma di una "piuma" di plasma che si espande verso il substrato, sul quale si forma il film. Si tratta di una tecnica di deposizione molto versatile che permette di produrre film e nanostrutture di vari materiali e morfologie al variare di diversi parametri di processo, quali l'energia degli impulsi, il numero totale di impulsi sul bersaglio, la loro frequenza di ripetizione, il gas all'interno della camera di deposizione e la sua pressione. Grazie a tale versatilità, si può applicare la PLD alla sintesi di materiali 2D su scala del centimetro, utilizzando temperature di crescita relativamente basse $\left(400-600^{\circ} \mathrm{C}\right)$ e fornendo un ottimo grado di controllo sul numero di strati atomici e sulla stechiometria dei film prodotti [9].

\section{OSSERVARE I MATERIALI 2D: TECNICHE DI CARATTERIZZAZIONE}

Una volta sintetizzati, è possibile "osservare" i materiali 2D tramite varie tecniche sperimentali, in grado di rivelarne le caratteristiche morfologiche, strutturali, elettroniche, ottiche, elettriche, magnetiche, chimiche, ecc. In generale, vista la natura bi-dimensionale dei materiali da studiare, risultano particolarmente efficaci le tecniche di caratterizzazione proprie della Scienza delle Superfici, che offrono la possibilità di "sondare" con sufficiente sensibilità pochi strati atomici. Alcune tra queste tecniche forniscono informazioni sulle proprietà strutturali: ad esempio, la microscopia elettronica a trasmissione (TEM) e le microscopie a scansione di sonda (SPM) permettono di osservare direttamente il reticolo cristallino con elevata risoluzione spaziale (nanometrica e subnanometrica), mentre tecniche di diffrazione quali la diffrazione di elettroni a bassa energia (LEED) e la diffrazione superficiale di raggi $X$ (SXRD) consentono di analizzare la struttura del reticolo reciproco. La struttura geometrica del reticolo atomico che costituisce il materiale contribuisce in maniera fondamentale a determinarne la struttura elettronica. Quindi è importante poter disporre di tecniche in grado di rivelare sia le caratteristiche strutturali sia quelle elettroniche. La microscopia a effetto tunnel (STM), capostipite delle tecniche SPM, offre questa possibilità, in quanto permette di osservare la superficie del materiale con risoluzione atomica e di rivelarne le proprietà elettroniche locali. Questa tecnica fu inventata nel 1981 da Gerd Binnig and Heinrich 
Röhrer, che vennero per questo insigniti del premio Nobel per la Fisica nel 1986 [10]. Il principio di funzionamento di questa tecnica si basa sull'effetto tunnel, noto effetto quantistico per cui gli elettroni hanno probabilità non nulla di attraversare una barriera di potenziale tra due elettrodi, se posti a una distanza sufficientemente piccola tra loro (dell'ordine di $1 \mathrm{~nm}$ ). Nel microscopio a effetto tunnel i due elettrodi sono una sottile punta metallica e la superficie del campione: una differenza di potenziale tra i due (dell'ordine di $1 \mathrm{~V}$ ) determina il passaggio di una corrente di tunnel (dell'ordine di $1 \mathrm{nA}$ ) che dipende sia dalla distanza punta-campione sia dalle proprietà elettroniche superficiali. $\mathrm{La}$ "scansione" della punta sulla superficie e la simultanea misura della corrente di tunnel permettono di ottenere, come risultato della misura STM, un'immagine della superficie caratterizzata da elevatissima risoluzione spaziale (fino a raggiungere la risoluzione atomica) e sensibile alla struttura elettronica locale. In particolare, la sensibilità agli stati elettronici superficiali può essere maggiormente sfruttata grazie alla tecnica di spettroscopia a effetto tunnel (STS), tecnica figlia della STM, che consente di ottenere uno spettro della densità locale di stati elettronici (LDOS) nell'intorno del livello di Fermi, fornendo così informazioni di carattere locale sulle proprietà elettroniche del materiale. Questa tecnica è complementare ad altre tecniche di spettroscopia elettronica comunemente utilizzate, come la spettroscopia di fotoemissione. Altre tecniche spettroscopiche, quali la spettroscopia Raman e la spettroscopia di fotoluminescenza (PL), consentono di studiare, rispettivamente, le proprietà vibrazionali e ottiche dei materiali 2D. La spettroscopia Raman rivela gli stati vibrazionali del materiale e risulta essere molto sensibile al numero di strati atomici di cui è composto un materiale 2D. La spettroscopia di fotoluminescenza misura a varie lunghezze d'onda la luce riemessa dal materiale in seguito ad un'eccitazione ottica, e si è dimostrata molto efficace nello studio delle proprietà optoelettroniche dei TMD, consentendo di osservare la transizione da semiconduttore a gap indiretto a diretto che caratterizza questi materiali al limite 2D.

\subsection{Osservazioni STM di alcuni materiali 2D}

In questa sezione verranno presentati alcuni risultati ottenuti da studi STM effettuati su tre materiali 2D: l'ossido di zinco $(\mathrm{ZnO}) 2 \mathrm{D}$, l'ossido di titanio $\left(\mathrm{TiO}_{\mathrm{x}}\right) 2 \mathrm{D}$ e il singolo strato di $\mathrm{MoS}_{2}$. Tali studi sono stati realizzati presso il Laboratorio Materiali Micro e Nanostrutturati 
del Politecnico di Milano. Il primo esempio riguarda lo $\mathrm{ZnO}$, uno dei materiali ossidi più studiati e utilizzati in numerose applicazioni. La sua forma bulk più stabile ha una struttura di tipo wurtzite, in cui ogni atomo di $\mathrm{Zn}(\mathrm{O})$ è legato a quattro atomi di $\mathrm{O}(\mathrm{Zn})$ in configurazione tetraedrica. Recentemente, studi teorici [11], poi confermati sperimentalmente, hanno dimostrato che, al limite $2 \mathrm{D}$, la struttura più stabile non è più quella wurtzitica, bensì una struttura di tipo grafite, in cui gli atomi di $\mathrm{Zn}$ e $\mathrm{O}$ si dispongono a nido d'ape in piani atomici perfettamente planari. La Fig. 1(a) mostra un'immagine STM in cui è possibile osservare dei nanocristalli di $\mathrm{ZnO} 2 \mathrm{D}$, prodotti tramite PLD su una superficie di oro. Grazie all'elevata risoluzione spaziale, sia verticale che laterale, del microscopio STM è possibile determinare lo spessore dei nanocristalli e, quindi, il numero di strati atomici che li compone. In particolare, in Fig. 1(b), si possono osservare due nanocristalli composti da uno e due strati atomici. Le immagini STM in Fig. 1 $(c, d)$ mostrano la superficie di questi nanocristalli a risoluzione atomica. Da immagini come queste è possibile osservare direttamente il reticolo atomico dello $\mathrm{ZnO} 2 \mathrm{D}$, caratterizzato da simmetria esagonale e da una cella unitaria di circa $0.32 \mathrm{~nm}$ [12]. Per quanto detto, lo $\mathrm{ZnO}$ è un esempio evidente di come al limite $2 \mathrm{D}$ i materiali possano presentare strutture nuove rispetto a quelle note in forma bulk. Un ulteriore esempio di questa proprietà generale è fornito dall'ossido di titanio $\left(\mathrm{TiO}_{\mathrm{x}}\right)$. Al limite $2 \mathrm{D}$, questo materiale forma strutture molto diverse rispetto a quelle bulk (rutilo e anatasio) in seguito al confinamento dimensionale e all'interazione con il substrato sul quale sono sintetizzate. Ad esempio, le immagini STM in Fig. 2(a,b) mostrano una particolare struttura del $\mathrm{TiO}_{\mathrm{x}}$ simile a quella del grafene, indotta dalla simmetria esagonale della superficie (111) dell'oro. Il modello strutturale di questa fase, ottenuto da un'analisi teorica, è raffigurato in Fig. 2(c,d) e mostra la disposizione a nido d'ape degli atomi di Ti e $\mathrm{O}$, che formano un singolo strato atomico [13]. Il terzo esempio, mostrato in Fig. 3, si riferisce al singolo strato di $\mathrm{MoS}_{2}$, uno dei materiali 2D più studiati. L'immagine STM in Fig. 3(a) mostra un tipico nanocristallo formato da un solo strato di $\mathrm{MoS}_{2}$ sulla superficie (111) dell'oro. La caratteristica più evidente della superficie del $\mathrm{MoS}_{2}$ è la presenza di una modulazione periodica esagonale, che si può meglio osservare in Fig. 3(b), insieme al reticolo atomico. Tale modulazione può essere interpretata prendendo in prestito un concetto proprio dell'Ottica: la figura (o pattern) di moiré. Questa si forma quando due 
reticoli aventi passo reticolare o orientazione diversi vengono sovrapposti. Nel nostro caso i due reticoli sono il reticolo atomico del $\mathrm{MoS}_{2}$ sovrapposto al reticolo atomico dell'oro. Come mostrato nella simulazione di Fig. 3(c), tale sovrapposizione dà luogo a un super-reticolo esagonale che descrive la modulazione periodica osservata nelle immagini STM [14]. La formazione di un pattern di moiré non è una caratteristica esclusiva del $\mathrm{MoS}_{2}$ su $\mathrm{Au}(111)$, ma si osserva comunemente in molti sistemi composti da materiali $2 \mathrm{D}$, dove può indurre modifiche nella struttura elettronica del materiale.
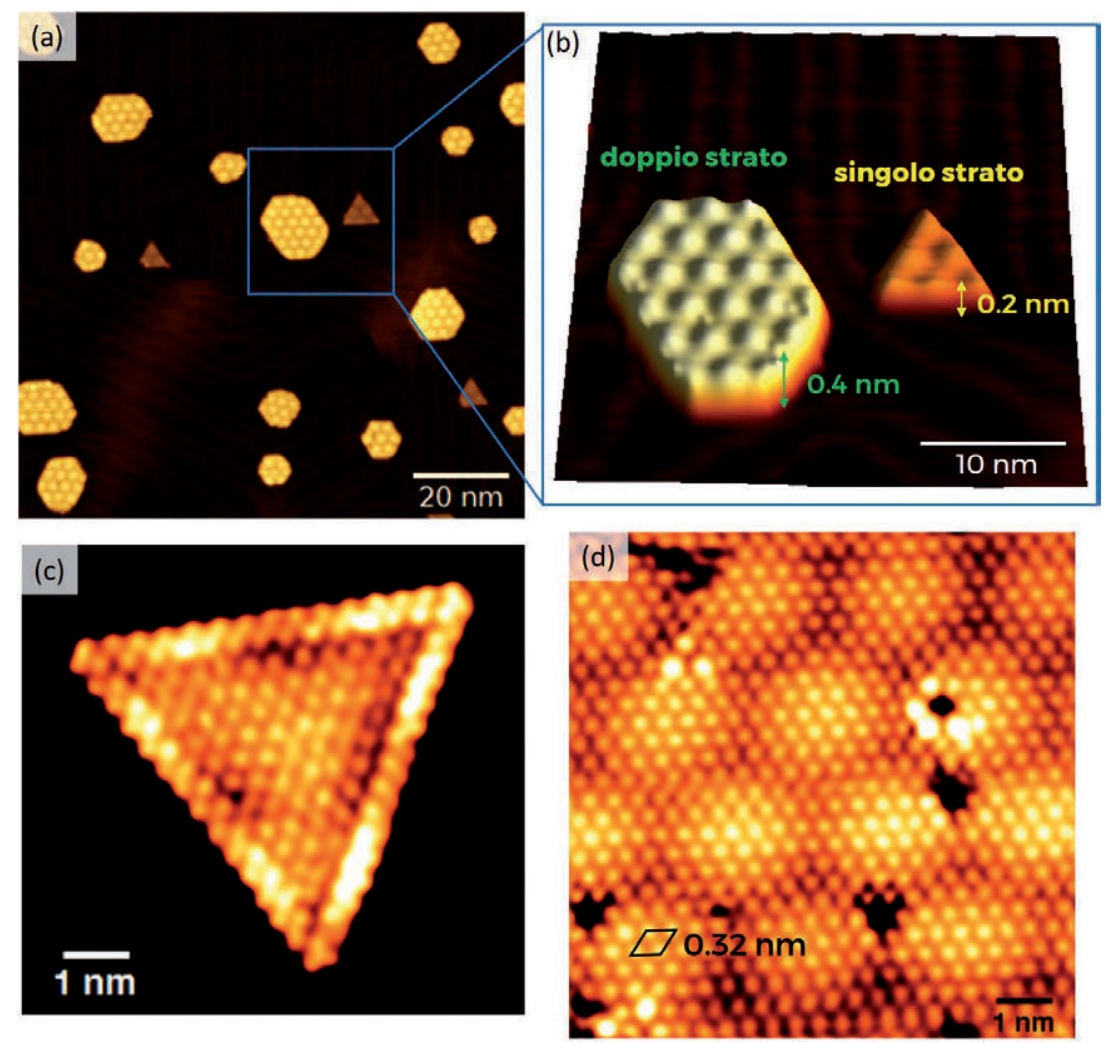

Fig. 1 - Immagini STM di nanocristalli di $\mathrm{ZnO} 2 \mathrm{D}$ sintetizzati tramite PLD sulla superficie (111) dell'oro. (a) Immagine STM di 200x200 $\mathrm{nm}^{2}$. (b) Immagine STM tri-dimensionale di due nanocristalli di cui è indicato lo spessore misurato rispetto al substrato. Quello più sottile è composto da uno strato atomico, mentre l'altro da due. (c)-(d) Immagini STM a risoluzione atomica della superficie di due nanocristalli: la cella unitaria dello $\mathrm{ZnO}$ misura $0.32 \mathrm{~nm}$. 


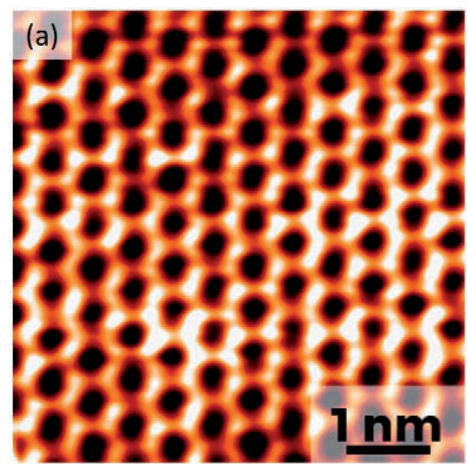

(c)

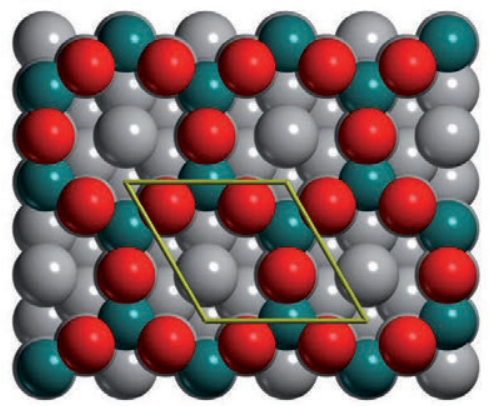

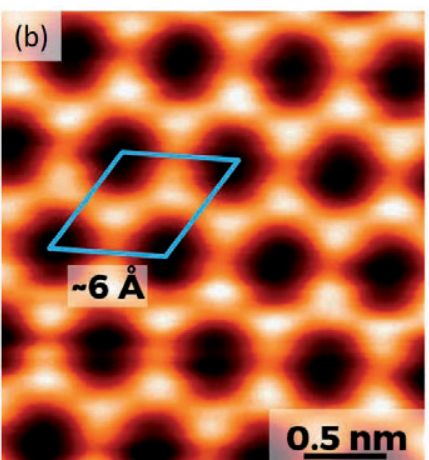

(d)

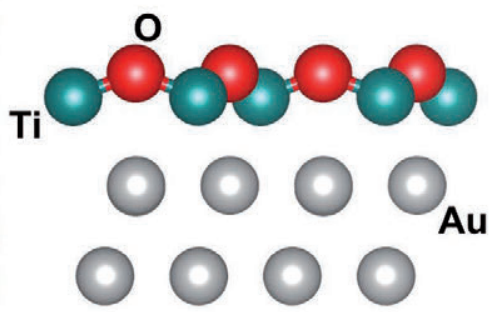

Fig. 2 - (a) Immagine STM della struttura a nido d'ape del TiOx 2D su Au(111). (b) Immagine STM a risoluzione atomica della stessa struttura. (c)-(d) Vista dall' alto e laterale del modello strutturale ottenuto da simulazioni teoriche. Legenda: rosso = ossigeno, verde = titanio, grigio $=$ oro.
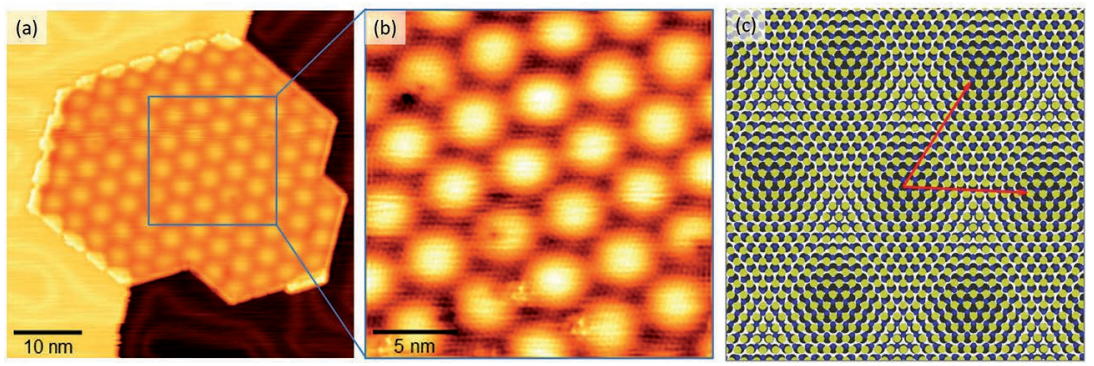

Fig. 3 - (a) Immagine STM di un nanocristallo formato da un singolo strato di MoS2 sintetizzato tramite PLD su Au(111). (b) Immagine STM a risoluzione atomica della superficie di MoS2, in cui si osserva il pattern di moiré esagonale. (c) Modello atomistico della sovrapposizione del reticolo di MoS2 su quello di oro: $i$ due vettori (in rosso) generano il super-reticolo che descrive la geometria del pattern di moiré. 


\section{CONCLUSIONI}

I materiali 2D sono materiali solidi ultrasottili, dello spessore al più di qualche strato atomico. Capostipite di questa classe di materiali è il grafene, il cui studio, a partire dal 2004, ha dato il via alla ricerca sui materiali $2 \mathrm{D}$ e lo loro uniche proprietà. Tali proprietà differiscono da quelle dei comuni materiali formati da molti strati atomici, per via di effetti fisici legati principalmente al confinamento bi-dimensionale. La ricerca sulle proprietà fondamentali e sui metodi di fabbricazione di questi materiali è in continua crescita e promette di aprire nuovi scenari di sviluppo tecnologico basati sulla manipolazione su scala nanometrica di materiali economici e facilmente reperibili. Tale sviluppo potrà interessare diversi ambiti applicativi, ma specialmente quello elettronico e optoelettronico, dove i limiti fisici imposti alla miniaturizzazione dei dispositivi rendono necessario concepire tecnologie alternative per realizzare sistemi sempre più avanzati. Oltre l'interesse applicativo, è importante sottolineare come lo studio di questi materiali ci metta a confronto con l'osservazione, l'analisi e la comprensione di proprietà e comportamenti fisico-chimici ancora sostanzialmente inesplorati. La ricerca sui materiali 2D segna, pertanto, un passaggio importante nella capacità dell'uomo di manipolare ed "ingegnerizzare" la materia su scala nanometrica, sulla quale si baserà lo sviluppo delle future nanotecnologie.

\section{RINGRAZIAMENTI}

Si ringrazia il Laboratorio Materiali Micro e Nanostrutturati del Dipartimento di Energia del Politecnico di Milano, presso il quale sono stati ottenuti i risultati sperimentali presentati.

\section{BIBLIOGRAFIA}

1. Geim AK, Novoselov KS. The rise of graphene. Nature Materials, 2007: 6(3): 183-191.

2. Butler SZ, et al. Progress, challenges, and opportunities in two-dimensional materials beyond graphene. ACS Nano, 2013: 7(4): 2898-2926.

3. Fiori G, Bonaccorso F, Iannaccone G, Palacios T, Neumaier D, Seabaugh A, Banerjee SK, Colombo L. Electronics based on two-dimensional materials. Nature Nanotechnology, 2014: 9(10): 768-779. 
4. Xia F, Wang H, Xiao D, Dubey M, Ramasubramaniam A. Two-dimensional material nanophotonics. Nature Photonics, 2014: 8(12): 899-907.

5. Wang QH, Kalantar-Zadeh K, Kis A, Coleman JN, Strano MS. Electronics and optoelectronics of two-dimensional transition metal dichalcogenides. Nature Nanotechnology, 2012: 7(11): 699-712.

6. Pacchioni G. Two dimensional oxides: Multifunctional materials for advanced technologies. Chemistry-A European Journal, 2012: 18: 10144-10158.

7. Geim AK, Grigorieva IV. Van der Waals heterostructures. Nature, 2013: 499: 419-425.

8. Novoselov KS, Jiang D, Schedin F, Booth TJ, Khotkevich VV, Morozov SV, Geim AK. Two-dimensional atomic crystals. Proceedings of the National Academy of Sciences of the United States of America, 2005: 102(30): 10451-10453.

9. Yang Z, Hao J. Progress in pulsed laser deposited two-dimensional layered materials for device applications. Journal of Materials Chemistry C, 2016: 4: 8859-8878.

10. Binnig G, Rohrer H, Gerber C, Weibel E. Surface studies by scanning tunneling microscopy. Physical Review Letters, 1982: 49(1): 57-61.

11. Freeman CL, Claeyssens F, Allan NL, Harding JH. Graphitic nanofilms as precursors to wurtzite films: theory. Physical Review Letters, 2006: 96(6): 066102.

12. Tumino F, Casari CS, Passoni M, Bottani CE, Li Bassi A. Pulsed laser deposition of two-dimensional $\mathrm{ZnO}$ nanocrystals on $\mathrm{Au}$ (111): growth, surface structure and electronic properties. Nanotechnology, 2016: 27(47): 475703.

13. Tumino F, Carrozzo P, Mascaretti L, Casari CS, Passoni M, Tosoni S, Bottani $\mathrm{CE}$, Li Bassi A. Two-dimensional TiOx nanostructures on $\mathrm{Au}$ (111): a scanning tunneling microscopy and spectroscopy investigation. 2D Materials, 2015: 2(4): 045011.

14. Tumino F, Casari CS, Passoni M, Russo V, Li Bassi A. Pulsed laser deposition of single-layer $\mathrm{MoS} 2$ on $\mathrm{Au}(111)$ : from nanosized crystals to large-area films. Nanoscale Advances, 2019: 1: 643-655. 
